\title{
The Utility of Positron Emission Tomography Scanning in Parkinson's Disease
}

\author{
Dr. Jason Seewoodhary ${ }^{1}$, \\ B.Sc (Hons) MBBCh (Hons) MRCP (UK) M.Sc (Dist) - Specialist Registrar in Diabetes Mellitus \& Endocrinology \\ and General Internal Medicine, Glan Clwyd Hospital, Rhyl, Denbighsire \\ UK LL18 5UJ \\ Dr. Stephen Wong MD FRCP (UK) ${ }^{2}$ \\ Consultant in Diabetes Mellitus \& Endocrinology and General Internal Medicine, Glan Clwyd Hospital, Rhyl, \\ Denbighsire, UK LL18 5UJ
}

\begin{abstract}
Positron Emission Tomography (PET) can visualise progression of Parkinsonian pathophysiology from early- through to end-stage disease generating objective quantitative longitudinal data, which enables assessing responses to treatment. The utility of PET scanning in the management of Parkinson's Disease is further exemplified by: refining the differential diagnosis of Parkinson's Disease as a distinct neurochemical entity; monitoring the efficacy of foetal nigral tissue transplantation; assessing the effects of surgical interventions such as deep brain stimulation; and guiding clinical management by studying the effects of drug-induced adverse effects. This concise review critically considers the role of PET scanning in the management of Parkinson's Disease.
\end{abstract}

Keywords: PET Scan; Parkinson's Disease; Stem Cell Therapy; Radioactive Tracers; Dopamine Receptors

Parkinson's disease (PD) is a progressive neurodegenerative disorder characterised by loss of pigmented A9 dopaminergic neurons within the ventral region of the substantia nigra pars compacta. These neurons project via $D_{1}$ receptors (direct pathway) and inhibitory $\mathrm{D}_{2}$ receptors (indirect pathway) to the putamen and caudate nuclei and regulate movement. The degeneration within the nigrostriatal system with associated pre- and post-synaptic neuronal dysfunction, including defects in dopamine metabolism, enzymology e.g. AADC, transporter proteins e.g. DAT, vesicular transport e.g. VMAT2, and receptors e.g. $\mathrm{D}_{1}$ and $\mathrm{D}_{2}$, can be functionally and quantitatively assessed by Positron Emission Tomography (PET) scanning. PET is a very sensitive and specific non-invasive nuclear imaging technique that uses a positron-emitting radionuclide tracer to generate three-dimensional pictures of functional processes such as blood flow, oxygen consumption and metabolic activity in vivo. This summary will critically consider the usefulness of PET imaging in the study of PD.

PET can visualise progression of Parkinsonian pathophysiology from early- through to end-stage disease generating objective quantitative longitudinal data, which enables assessing responses to treatment. This contrasts favourably with post-mortem studies, which are limited in their application only providing 'snap-shot' data on advanced disease. Using 18F-DOPA PET imaging can quantify the rate and extent of nigrostriatal dopaminergic degeneration; this positively correlates with clinical data obtained from rating scales such as UPDRS ${ }^{1}$. This can be used to screen for pre-clinical lesions in asymptomatic at-risk relatives of patients with familial PD and patients with isolated tremor.

PET imaging usefully guides clinical management in PD. PET has shown that hypokinesia is due to failure to activate the supplementary motor and dorsal pre-frontal cortex ${ }^{2}$, with dopaminergic activity restored towards

Normality by dopamine agonists, monoamine oxidase inhibitors and amantadine. This is illustrated in figure 1. PET imaging allows studying drug-induced adverse effects in vivo, for example, in determining the occupancy rate of striatal $\mathrm{D}_{2}$ receptor antagonism by antipsychotics in PD patients with neuropsychiatric symptoms; this can aid selection of novel atypical antipsychotics that do not exacerbate Parkinsonian symptoms ${ }^{3}$.The usefulness of PET in imaging $\mathrm{D}_{2}$ receptors is further exemplified by refining the differential diagnosis of PD as, in part, a distinct

\footnotetext{
${ }^{1}$ Corresponding Author: seewoodharyj@hotmail.com
} 


\section{American Research Journal of Medicine and Surgery, Volume 1, Issue 1, 2015}

ISSN 2379-8955

neurochemical entity separate from Progressive Supranuclear Palsy, Corticobasal Degeneration and Multiple Systems Atrophy ${ }^{4}$.PET scanning has been used to monitor the efficacy of foetal nigral tissue transplantation in PD; evidence from a double-blind controlled trial concluded that transplants of foetal tissue cannot be recommended due to an association with dyskinesias ${ }^{5}$. PET has also been used to assess the effects of surgical interventions such as pallidotomy and deep brain stimulation in $\mathrm{PD}^{6}$.

The usefulness of PET in the study of PD is offset by limitations including: rapid decay of radioactive tracers restricting use to monitoring short tasks; use of ionising radiation; high costs; relatively low spatial resolution; and lengthy scan times causing discomfort.

In summary, by providing dynamic insights into functional pathology, PET has revolutionised the clinical management of PD.

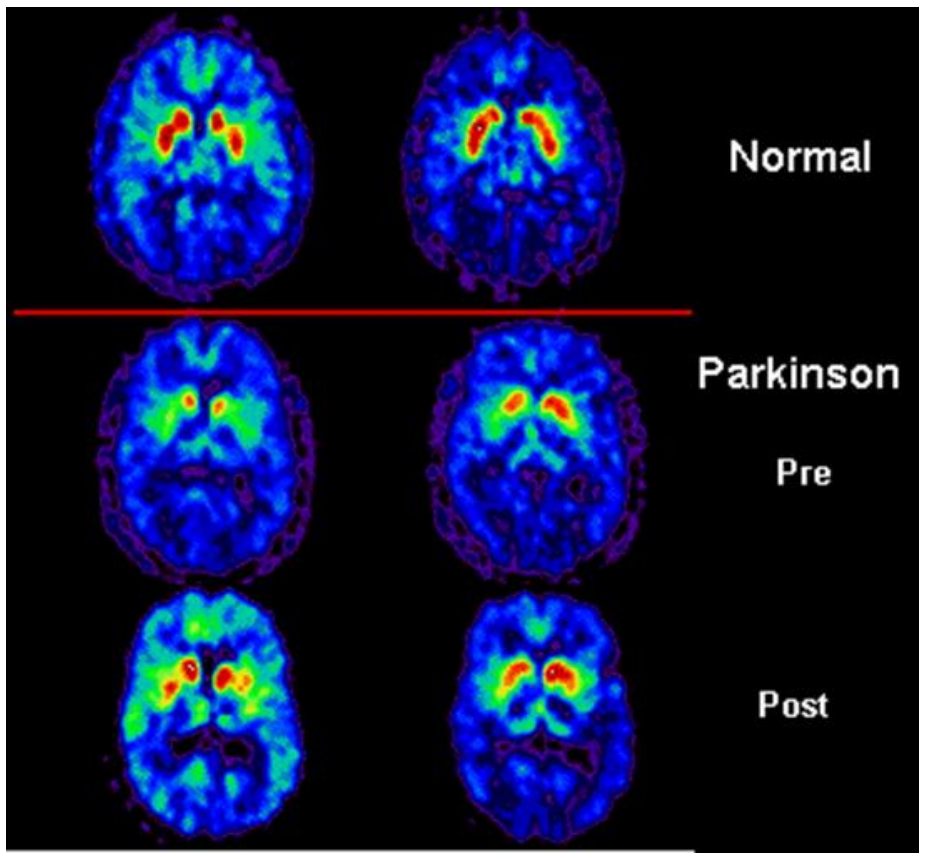

Fig1. The images show the concentration of dopaminergic nigrostriatal neurons; warmer colours represent a higher concentration. Comparing images of a healthy patient (top) and a PD patient (bottom), shows a decrease in PET signal (red colour) in the PD brain prior to treatment, which increases after dopaminergic therapy. (Imported from htt/www.wikidoc.org/index.php/Parkinson's_disease_other_imaging_findin

\section{REFERENCES}

[1] Karimi M, Golchin N et al., Subthalamic nucleus stimulation-induced regional blood flow responses correlate with improvement of motor signs in Parkinson disease. Brain 131 (2008): pp 2710-19.

[2] Fukuda M, Edwards C et al., Functional brain networks in Parkinson's disease. Parkinsonism Relat. Disord. 8 (2001): pp 914.

[3] Brucke T, Djamshidian S et al., SPECT and PET imaging of the dopaminergic system in Parkinson's disease. J Neurol. 247 (2000): Suppl 4: IV/2-7.

[4] Brooks DJ, Ibanez V et al., Differing patterns of striatal 18F-dopa uptake in Parkinson's disease, multiple system atrophy, and progressive supranuclear palsy. Ann. Neurol. 28 (1990): pp 547-555.

[5] Olanow CW, Goetz CG et al., A double-blind controlled trial of bilateral fetal nigral transplantation in Parkinson's disease. Ann Neurol. 54 (2003): pp 403-14.

[6] Mure H, Tang CC et al., Improved sequence learning with subthalamic nucleus deep brain stimulation: evidence for treatment-specific network modulation. The Journal of Neuroscience 32 (2012): pp 2804-13. 